\title{
O absenteísmo da equipe de enfermagem em unidade de pronto socorro de um hospital universitário ${ }^{1}$
}

\section{Absenteeism in the nursing team at the emergency unit of a university hospital}

\author{
El absentismo del equipo de enfermería en unidad de urgencias en el hospital universitario
}

\author{
Elisângela Cristina de Campos', Carmen Maria Casquel Monti Juliani", Valéria Castilho Palhares ${ }^{\prime \prime \prime}$ \\ ${ }^{1}$ Projeto de iniciação cientifica financiado pela fundação de Amparo à pesquisa do estado de São Paulo-FAPESP. \\ ' Enfermeira graduada pela Universidade Estadual Paulista (UNESP), Botucatu-SP. Email: elisangela.campos5@gmail.com. \\ "Profa Dra da disciplina de Administração Aplicada à Enfermagem da UNESP do Departamento de Enfermagem da Faculdade de Medicina \\ Campus de Rubião Junior. Email: cjuliani@fmb.unesp.br. \\ "I' Enfermeira. Chefe da Seção Técnica de Adultos do Pronto Socorro do Hospital das Clínicas de Botucatu/SP. Email: valeria@fmb.unesp.br.
}

\section{RESUMO}

O absenteísmo ou ausentismo é o termo utilizado para caracterizar as ausências dos funcionários ao trabalho. Essas ausências são definidas em previstas e não previstas. Para este estudo especificamos as ausências previstas como folgas, feriados e férias e para as não previstas ficam as faltas, licenças medicas, nojo e outras licenças. O objetivo foi investigar o real numero de ausências dos técnicos e auxiliares de enfermagem de um Pronto Socorro de um Hospital Universitário do interior do estado de São Paulo para definir o Índice de Segurança Técnica-IST. Foi utilizada metodologia quantitativa descritiva, e a coleta de dados feita de janeiro de 2006 a junho de 2007, a partir de arquivos administrativos após autorização do serviço. Entre os principais achados encontramos um IST cuja média gira em torno de 51\%. Concluímos que, embora próximo, está ligeiramente superior ao encontrado em outra realidade de Hospital Universitário. Entre as ausências não previstas, destacam-se as licenças médicas, que podem estar relacionadas à sobrecarga da equipe.

Descritores: Absenteísmo; Enfermagem; Recursos humanos.

\section{ABSTRACT}

Absenteeism is the term used to describe absences from work by employees. These absences are defined as planned and unplanned. For this specific study, planned absences were days in lieu, statutory holidays, and vacations, and unplanned were missed days, medical leave, sickness, and other leaves. The aim was to investigate the actual number of absences by technicians and nursing auxiliaries in a University Hospital emergency unit in São Paulo state to define a Technical Safety Index - IST. Quantitative descriptive methodology was used, and data were collected since 2006, January until 2007, June from hospital records after authorization from the service. One of the main findings was that IST was about $51 \%$. We conclude that, although close, it is slightly higher than found in another reality of University Hospitals. From the unplanned absences, medical leave stood out; this could be related to team overload.

Descriptors: Absenteeism; Nursing; Human resources.

\section{RESUMEN}

El absentismo o ausentismo es el término utilizado para caracterizar las ausencias de los empleados al trabajo. Esas ausencias son divididas en previstas y no previstas. Para este estudio especificamos las ausencias previstas como días libres, feriados y vacaciones y para las no previstas quedan las faltas, licencias médicas, luto y otros permisos. El objetivo fue investigar el real número de ausencias de los técnicos y auxiliares de enfermería de una sección de urgencias de un hospital universitario en el estado de São Paulo para definir el Índice de Seguridad Técnica-IST. Fue utilizada metodología cuantitativa descriptiva y la cosecha de datos fue hecha de enero 2006 hasta junio de 2007 con archivos administrativos tras autorización del servicio. Entre los principales hallazgos encontramos un IST cuyo promedio gira alrededor del $51 \%$. Llegamos a la conclusión de que, aunque estrecha, es ligeramente superior a lo encontrado en otra realidad de hospital universitario. Entre las ausencias no previstas se destacan las licencias médicas, que pueden estar relacionadas con la sobrecarga del equipo.

Descriptores: Absentismo; Enfermeria; Recursos humanos. 


\section{NTRODUÇÃO}

O absenteísmo ou absentismo é o termo utilizado para caracterizar a ausência do funcionário ao trabalho. De forma mais abrangente, é o somatório das faltas dos trabalhadores, não sendo essas decorrentes de desemprego, doença prolongada ou licença legal ${ }^{(1)}$.

O Conselho Federal de Enfermagem desmembra essas faltas em ausências planejadas, como, folgas, férias e feriados e as não planejadas, que incluem faltas, licenças médicas, acidentes, entre outras ${ }^{(2)}$.

$\mathrm{Na}$ enfermagem o absentismo deve ser um ponto importante a ser considerado e analisado no gerenciamento de pessoal, em especial nos serviços que funcionam 24 horas. Dados de absenteísmo refletem no dimensionamento de pessoal de enfermagem, em especial em Instituições complexas, como é a hospitalar, uma vez que esses estudos são considerados nesses cálculos.

Além disso, o percentual de ausências de outros estudos chamam atenção sobre a arbitrariedade em se utilizar índices de cobertura indicados na literatura, tendo em vista a disponibilidade de métodos que possibilitam a identificação desses valores para cada realidade ${ }^{(3)}$.

Nesse contexto, se faz necessário pensar no ausentismo não somente em seu quantitativo, mas também nos fatores desencadeadores dessas faltas.

Em estudo, os autores elencaram os seguintes fatores: necessidade de dupla jornada de trabalho, a fim de suprir o déficit salarial; funcionários casados em com filhos que ainda não estão na idade escolar; tipo de função desempenhada; insalubridade; problemas ergonômicos; tensão emocional; cansaço físico e mental ${ }^{(4)}$. Ainda nessa vertente, é aponta como uma possível causa de absenteísmo, a questão de o funcionário ser estatutário, a qual the confere estabilidade no emprego ${ }^{(5)}$.

A sobrecarga a que os profissionais estão expostos tem retratado um quadro de doenças ocupacionais como, distúrbios na alimentação, no sono, estresse e desorganização familiar ${ }^{(6)}$.

As doenças com maiores incidências são as relacionadas com 0 aparelho respiratório, geniturinárias, órgãos dos sentidos, sistema digestório e aparelho reprodutor feminino. Para as pesquisadoras, essas patologias podem estar diretamente relacionadas com as condições de trabalho, devido aos riscos que esses profissionais estão expostos ${ }^{(7)}$.

Diante da problemática do absenteísmo e da complexidade de uma instituição de saúde, o gerente de enfermagem tem em suas mãos um desafio na questão administrativa, em especial nos serviços que funcionam 24 horas e, portanto, dependem da continuidade da assistência para o sucesso do trabalho ${ }^{(8)}$.
O absenteísmo, embora não seja a única questão que interfere com a qualidade do cuidado, é um das variáveis a serem analisadas na avaliação do padrão de cuidado.

As ausências na enfermagem desorganizam o trabalho da equipe, pois um membro ausente gera perturbações e sobrecarga aos demais trabalhadores do grupo $^{(9)}$.

O absenteísmo de técnicos e auxiliares representa um grande desafio para a enfermagem gerencial, pois precisa garantir que o indivíduo seja atendido em todas as suas necessidades ${ }^{(10-11)}$. O dimensionamento de pessoal de enfermagem é definido como "etapa inicial do processo de provimento de pessoal que tem por finalidade a previsão da quantidade de funcionários por categoria, requerida para suprir as necessidades de assistência de enfermagem, direta ou indiretamente prestada ao cliente" $^{\prime(12)}$.

O cuidado de enfermagem de qualidade está diretamente relacionado com os recursos humanos, que devem ser quantitativamente e qualitativamente adequados $^{(13)}$.

O comprometimento do número ideal de funcionários repercute na assistência e eleva os custos para as instituições ${ }^{(14)}$. Dessa forma uma alocação correta de funcionários por meio de dimensionamento de pessoal, auxilia as organizações de saúde a adequar os seus custos, dados que estes são cada vez mais escassos, melhorar a qualidade da assistência e implementar novos métodos de cuidados $^{(15)}$.

A não previsão de quantidade e qualidade do pessoal compromete diretamente a qualidade do cuidado. O ausentismo é um problema de natureza complexa por envolver uma série de causas e, portanto de difícil controle, o que acaba por elevar os custos administrativos da instituição e prejuízos tanto para os pacientes quanto aos trabalhadores ligados ao cuidado(8,16). A fim de se minimizar o efeito danoso das ausências, tem sido feito o estudo do absenteísmo, que indica o índice de segurança técnico (IST) necessário para o dimensionamento de pessoal. Esse índice permite prever faltas como licença, folga, aprimoramento, casamento, dentre outras, não podendo, segundo Resolução do COFEN, ser inferior a 15\% do total. Nesta Resolução também, fica garantida a autonomia do enfermeiro para dimensionar e gerenciar o quadro de profissionais $^{(2,17)}$.

O absenteísmo tem sido apresentado num cenário que merece ser repensado, pois o pessoal de enfermagem fica como agente passivo das ações e marcado por condições de trabalho com características Tayloristas como: tarefas fragmentadas, trabalho hierarquizado, poder mínimo de decisão e insatisfação freqüentemente elevada no 
ambiente de atuação, refletindo assim nos altos índices de ausências e afastamentos por doenças ${ }^{(16)}$.

Preocupadas com essa questão, propusemos um estudo de absenteísmo de auxiliares e técnicos de enfermagem na Seção Técnica da Enfermaria de Adultos de uma Unidade de Pronto Socorro de um Hospital Escola do interior do estado de São Paulo, pois a problemática do absenteísmo percebido pela gerente do serviço apresentava-se relacionada a esses profissionais da equipe de enfermagem.

Essa pesquisa justificou-se por permitir um conhecimento da realidade das ausências de auxiliares e técnicos de enfermagem da Seção Técnica da Enfermaria de Adultos do Pronto Socorro e esses dados orientam o planejamento das ações gerenciais do profissional enfermeiro. Uma vez que o desconhecimento das reais taxas de absenteísmo, que podem variar de uma realidade para outra, dificultam o processo de planejamento e gestão dos recursos humanos de enfermagem, seu conhecimento permite melhorar a gestão e argumentação junto aos administradores, para a contratação e manutenção de um quadro de recursos humanos que possa promover uma assistência de qualidade aos clientes. Pode assim subsidiar as atividades de gerenciamento em enfermagem com o conhecimento do IST para nortear o dimensionamento de pessoal.

Assim, o objetivo da pesquisa foi investigar o real número de ausências dos técnicos e auxiliares de enfermagem de um Pronto Socorro de um Hospital Universitário do interior do estado de São Paulo para definir o Índice de Segurança Técnica-IST.

\section{METODOLOGI A}

Estudo exploratório e descritivo, de natureza quantitativa baseado em registros e análise dos dados coletados referentes às ausências de auxiliares e técnicos de enfermagem.

A pesquisa foi realizada em uma Seção Técnica de Enfermaria de Adultos do Pronto Socorro de um Hospital Universitário do interior do estado de São Paulo. O Hospital é público, atende à clientela do SUS e é referência para atendimentos mais complexos em sua região. Segundo dados do Centro de Informática Médica, o Hospital, em estudo, possui 366 leitos oficiais. No entanto, conta ainda com 140 leitos extras. Pela nossa experiência no Hospital, podemos afirmar que existe grande ocupação desses leitos, confirmado pelas taxas de ocupação que, em alguns serviços, excedem 100\%, devido ao fato deste serviço ser uma referência regional dentro do Sistema Único de Saúde. A Seção Técnica de Enfermaria de Adultos, que é foco desta pesquisa, conta com 22 leitos divididos em 09 leitos de UTI e 13 leitos de enfermaria.

Com relação aos recursos humanos, o referido serviço possuía em média 56 funcionários para o ano de 2006 e 52 para o ano de 2007. Dentre estes trabalhadores, $75 \%$ são auxiliares e $25 \%$ técnicos de enfermagem, dos quais $27,3 \%$ são contratos temporários, $50 \%$ contratados pela Fundação para o Desenvolvimento Médico e Hospitalar (Famesp) e $22,7 \%$ pela Unesp. Os funcionários da Unesp são os concursados pelo quadro funcional e regidos pelo Estatuto dos Servidores Públicos, os contratados pela Famesp são os aprovados em processo seletivo e contratados pelo regime da Consolidação das Leis do Trabalho (CLT) e, finalmente, os contratos temporários também são feitos pela mesma Fundação, a Famesp, porém por tempo determinado, em geral, 90 dias, para resolver situações emergenciais como, por exemplo, cobertura de licenças. Estes últimos não configuram vínculo empregatício.

A coleta de dados foi feita a partir de dados retrospectivos dos registros administrativos e gerenciais da Chefia de Enfermagem da referida Unidade. Para a organização dos dados, foi utilizada planilha em Excel, elaborada e cedida por Gaidzinski(12) para registro das ausências.

A planilha foi organizada registrando ausência em dias, portanto, quando os registros estavam em horas, estes foram divididos por $8 \mathrm{~h}$, correspondendo a um dia de trabalho no referido Hospital, que possui jornada de trabalho de $40 \mathrm{~h}$ semanais.

O período de coleta e organização dos dados foi de maio a outubro de 2007, no entanto, os dados referem-se ao período de janeiro de 2006 a junho de 2007.

Como base para os cálculos foi considerada neste trabalho a média de 56 funcionários entre auxiliares e técnicos de enfermagem, não participando desse quantitativo os enfermeiros dessa unidade.

Para efeito de cálculo, utilizou-se como definição de ausências previstas as folgas, as férias e os feriados e para as não previstas as faltas e as licenças (médicas, maternidade, acidentes, INSS, nojo, gala, suspensões e licenças prêmio), conforme proposta de Gaidzinski, Fugilin e Castilho ${ }^{(18)}$.

As equações utilizadas foram extraídas da proposta das mesmas autoras ${ }^{(18)}$.

- Folgas semanais (E\%):

$$
\mathrm{E} \%=\frac{e}{D-e} .100
$$

onde:

$\mathrm{E} \%=$ percentual de folgas.

e = numero de dias de folga, por semana, dos trabalhadores da enfermagem.

$\mathrm{d}=$ número de dias trabalhados na unidade.

- Ausências previstas por feriados (F\%):

$$
F \%=\frac{f}{D-f} .100
$$


onde:

$\mathrm{F} \%=$ percentual de dias de feriados.

$\mathrm{f}=$ dias de feriado no ano.

$\mathrm{D}=$ dias do ano, 365 dias.

- Ausências previstas por férias $\left(\mathrm{V}_{\mathrm{k}} \%\right)$ :

$$
\mathrm{V}_{\mathrm{k}} \%=\frac{v_{k}}{D-v_{k}} .100
$$

onde:

$\mathrm{V}_{\mathrm{k}} \%=$ percentual de férias, segundo a categoria profissional $_{k}$ (técnicos e auxiliares de enfermagem).

$\mathrm{v}_{\mathrm{k}}=$ média de dias de ferias categoria profissional $_{k}$.

$\mathrm{D}=$ dias do ano, 365 dias.
- Ausências não previstas $\left(A_{k} \%\right)$ :

$$
\mathrm{A}_{\mathrm{k}} \%=\left(\frac{\sum_{i} a_{k, i}}{D-\sum_{i} a_{k, i}}\right) \cdot 100
$$

onde:

$A_{k} \%=$ percentual de ausências não previstas, segundo a categoria profissional $k$ (técnicos $e$ auxiliares de enfermagem).

$\sum_{i} a_{k, i}=$ somatório de dias médios de ausências não previstas, segundo os tipos de ausências, por categoria de profissionais.

$\mathrm{D}=$ dias do ano, 365 dias.

- I Índice de Segurança Técnico (IST\%):

$$
\operatorname{IST} \%=\left\{\left[\left(1+\frac{E \%}{100}\right) \cdot\left(1+\frac{F \%}{100}\right) \cdot\left(1+\frac{V_{k}}{100}\right) \cdot\left(1+\frac{A_{k}}{100}\right)\right]-1\right\} .100
$$

A pesquisa não foi remetida ao Comitê de Ética e Pesquisa local, porque os dados provêm apenas de relatórios administrativos, não sendo, portanto, recomendada a análise pelo Comitê. Embora não tenha sido encaminhado ao Comitê, foi solicitada e obtida formalmente autorização do serviço para a coleta dos dados.

\section{RESULTADOS E DISCUSSÃO}

Considerando que no projeto inicial a coleta de dados seria limitada ao ano de 2006 e, posteriormente, o período foi ampliado, incluindo o primeiro semestre de 2007 para que pudesse haver uma comparação entre os dois anos, para avaliarmos se a tendência se mantinha, optamos então por apresentar separadamente os dados de 2006 e 2007.

Depois de organizados os dados, os mesmos foram analisados utilizando recursos da estatística descritiva, apresentada em números absolutos e relativos, gráficos e tabelas.

Inicialmente apresentaremos a proporção de ausências previstas e não previstas, demonstrando a representação percentual de cada tipo de ausência em relação ao total (Gráfico 1 e 2 e Tabela 1 e 2) para, posteriormente aplicar as fórmulas que levarão ao cálculo do índice de absenteísmo.

Gráfico 1: Percentual de ausências previstas e não previstas. Botucatu, 2006.

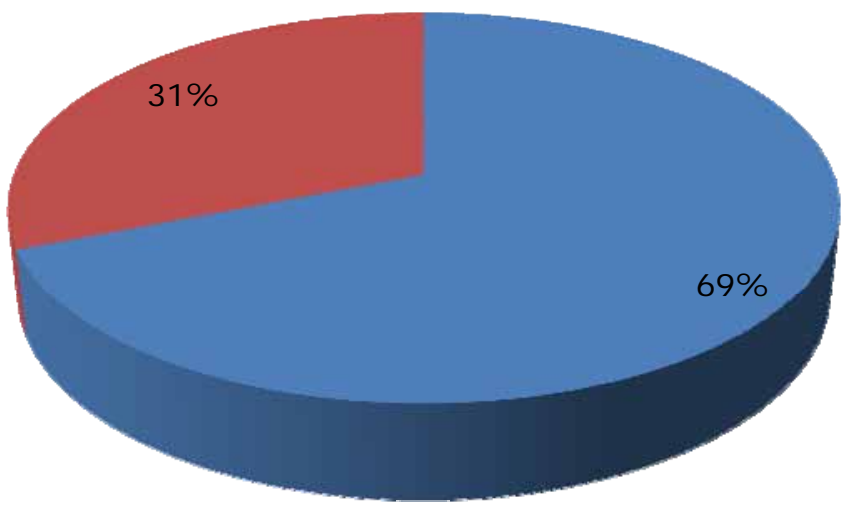

- Não prevista

- Prevista 
Gráfico 2: Percentual de ausências previstas e não previstas. Botucatu, 2007.

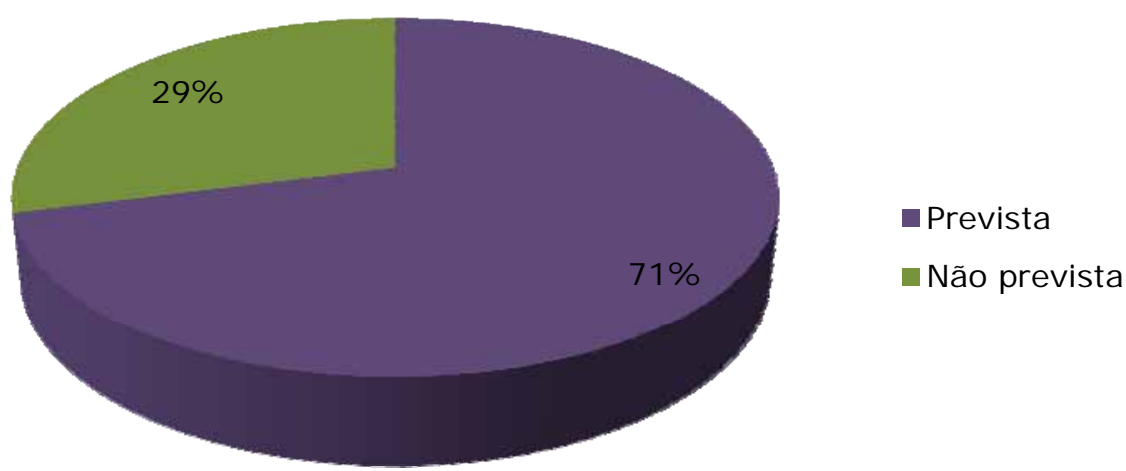

Tabela 1: Percentual por tipo de ausência prevista. Botucatu, 2006 e 2007.

\begin{tabular}{|c|c|c|c|c|}
\hline \multirow{2}{*}{ Tipo de ausência } & 2006 & \multirow{2}{*}{$\%$} & 2007* & \multirow{2}{*}{$\%$} \\
\hline & No & & No & \\
\hline Folgas & 4044 & 71,1 & 2005 & 72,4 \\
\hline Feriados & 567 & 10 & 299 & 10,8 \\
\hline Férias & 1073 & 18,9 & 465 & 16,8 \\
\hline TOTAL & 5684 & 100 & 2769 & 100 \\
\hline
\end{tabular}

* 2007 refere-se apenas ao primeiro semestre do ano.

Tabela 2: Percentual por tipo de ausência não prevista. Botucatu, 2006 e 2007.

\begin{tabular}{|c|c|c|c|c|}
\hline \multirow{2}{*}{ Tipo de ausência } & $\mathbf{2 0 0 6}$ & \multirow{2}{*}{$\mathbf{2 0 0 7}$} & $\mathbf{2}$ & \multirow{2}{*}{$\%$} \\
\cline { 2 - 2 } \cline { 4 - 5 } & $\mathbf{N o}$ & 1,58 & $\mathbf{N}$ & 2,98 \\
\hline Faltas & 40 & 82,41 & 981 & 88,77 \\
\hline Licenças Médicas & 2080 & 15,69 & 90 & 8,16 \\
\hline Licença Prêmio & 396 & 0,32 & 1 & 0,09 \\
\hline Nojo & 8 & $\mathbf{1 0 0}$ & $\mathbf{1 1 0 5}$ & $\mathbf{1 0 0 , 0}$ \\
\hline TOTAL & $\mathbf{2 5 2 4}$ &
\end{tabular}

* 2007 refere-se apenas ao primeiro semestre do ano.

Confirmando achado de estudo anterior $^{(3,9)}$ fica clara a importância das ausências previstas que é o grande percentual que representa a necessidade de cobertura de férias, folgas e feriados para que os serviços possam funcionar 24 horas ininterruptamente.

No ano de 2006, das 8208 ausências, 5684 $(69,3 \%)$ corresponderam às ausências previstas, sendo a maior fatia destas as folgas com 4044 $(49,2 \%)$ e $2524(30,7 \%)$ foram ausências não previstas, sendo dentro destas, as licenças médicas as mais significativas com $2080(25,3 \%)$, resultado corroborado pelo estudo realizado em $2007^{(9)}$.

Para o ano de 2007, apenas com os dados do primeiro semestre, observamos que a tendência se mantém, ou seja das 3874 ausências, 2769 (71,4\%) tratam-se das ausências previstas, entre estas sendo $2005(51,7 \%)$ para as folgas. Quanto às 1105 $(28,6 \%)$ ausências não previstas, as licenças médicas representam $981(25,3 \%)$ do total de ausências.

Até aqui, discutimos as proporções simples dos tipos de ausência nos períodos estudados. A partir de agora, aplicando as fórmulas já referidas, calcularemos os índices conforme proposto, para chegarmos ao cálculo do índice de absenteísmo (IST) do serviço.

Índices baseados nos dados de 2006. 


$$
\begin{array}{rlrl}
\mathrm{E} \% & =\frac{72}{365-72} \cdot 100 & \mathrm{E} \%=24,6 \\
\mathrm{~F} \%=\frac{10}{365-10} \cdot 100 & F \%=2,8 \\
\mathrm{~V}_{\mathrm{k}} \%=\frac{19}{365-19} \cdot 100 & \mathrm{~V}_{\mathrm{k}} \%=5,5 \\
\mathrm{~A}_{\mathrm{k}} \%=\left(\frac{45}{365-45}\right) \cdot 100 & \mathrm{~A}_{\mathrm{k}} \%=14 \\
\text { IST } \%=\left\{\left(\left(1+\frac{24,6}{100}\right) \cdot\left(1+\frac{2,8}{100}\right) \cdot\left(1+\frac{5,5}{100}\right) \cdot\left(1+\frac{14}{100}\right)\right]-1\right\} .100 & \mathrm{IST}^{2} \%=51,3
\end{array}
$$

No ano de 2006, atribuímos um percentual do IST de $24.6 \%$ referente às folgas semanais, as quais o trabalhador tem direito. Para efeito de calculo não consideramos apenas as folgas registradas em escala mensal, fizemos uma analise da carga horária mensal determinada e a carga horária mensal realmente trabalhada por funcionário. O mesmo fora feito para os feriados, e neste caso não foi considerado os feriados que coincidiram com licenças médicas, estes por fim perfizeram um total de $2.8 \%$, ficando para as férias um total de $5.5 \%$ ambos com valores abaixo do encontrado em estudo semelhante ${ }^{(17)}$. Esse índice mais baixo pode ser explicado pelo fato de parte dos funcionários, em especial os regidos pela CLT converterem parte das férias em pecúnia, deste modo os dias de férias remunerados e não usufruídos em descanso acabam por abaixar esse índice.

Conforme apontado anteriormente, dentre as ausências não previstas o maior parte foi atribuído às licenças médicas. Diante disto calculamos o IST total no valor de $51,3 \%$.

Em 2007 encontramos um quantitativo de ausências de 3874, ficando para as ausências previstas um total de 2769 . Para os cálculos abaixo utilizamos a média de ausências conforme indicado na fórmula, porém considerando o número de dias do primeiro semestre.

$$
\begin{array}{crl}
\mathrm{E} \% & =\frac{38,5}{182-38,5} \cdot 100 & \mathrm{E} \%=26,8 \\
F \%=\frac{5,8}{182-5,8} \cdot 100 & F \%=3,3 \\
\mathrm{~V}_{\mathrm{k}} \%=\frac{8,9}{182-8,9} \cdot 100 & \mathrm{~V}_{\mathrm{k}} \%=5,2 \\
\mathrm{~A}_{\mathrm{k}} \%=\left(\frac{18}{182-18}\right) \cdot 100 & \mathrm{~A}_{\mathrm{k}} \%=11 \\
\text { IST } \%=\left\{\left(\left(1+\frac{26,8}{100}\right) \cdot\left(1+\frac{3,3}{100}\right) \cdot\left(1+\frac{5,2}{100}\right) \cdot\left(1+\frac{11}{100}\right)\right]-1\right\} .100 & \mathrm{IST} \%=51,2
\end{array}
$$

Nestes cálculos, encontramos resultados de um IST de $51,3 \%$ para 2006 e $51,2 \%$ para 2007 , que não difere muito de estudo similar cujo achado revela um IST de $49 \%$ para técnicos e auxiliares em realidade semelhante, ou seja, hospital escola ${ }^{(9)}$. Após os cálculos apresentados encontramos uma taxa de $26.8 \%$ referente às folgas, $5.2 \%$ relacionadas às férias e $3.3 \%$ aos feriados, e para as ausências não previstas um total de $11 \%$ e deste total também a maioria atribuídas às licenças medicas, acompanhando a mesma tendência de 2006, demonstrando um IST total de $51,2 \%$ para 2007, considerando-se o primeiro semestre.
Os dados nos permitiram identificar que as ausências previstas, representando o dobro das não previstas nos dois períodos, vem sendo a principal responsável pela problemática da cobertura dos serviços que funcionam 24 horas e não deste serviço especificamente, ou seja, para que sejam cumpridos os direitos trabalhistas e a carga horária semanal respeitada, é necessário um significativo contingente de pessoas para essa cobertura. Essa reflexão é importante, pois o conceito de absenteísmo é, no senso comum, relacionado à falta ao serviço, mas neste caso, o conceito está além dela, representando o contexto no sentido de considerar todas as 
situações envolvidas para a garantia e continuidade do processo de trabalho que assegure assistência contínua e de qualidade ao cliente.

O número reduzido de trabalhadores na equipe de enfermagem compromete o funcionamento do serviço, atingindo diretamente a equipe e os pacientes, pois o ausentismo acarreta sobrecarga de trabalho na equipe e consequentemente uma assistência de enfermagem deficiente ${ }^{(9)}$.

Estudos sobre o estresse ocupacional vêm sendo realizados com a finalidade de se conhecer o impacto que a falta de materiais e o déficit de recursos humanos pode acarretar na vida dos trabalhadores bem como na prestação nos serviços de saúde ${ }^{(19-20)}$.

Diante dos achados deste estudo, faz-se necessário um olhar atento para as licenças médicas, que embora estejam em menor número dentro do total de ausências (previstas e não previstas), ainda se mostram com um percentual expressivo dentro das ausências não previstas. Comparado com outros estudos realizados em contexto semelhante, ou seja, em Hospital Universitário, encontramos em um deles $^{(4)}$ o índice de $63,2 \%$, em um outro ${ }^{(16)}$ que englobou quatro hospitais de ensino uma variação de 65 a $90 \%$ e, se considerarmos a média entre esses quatro hospitais temos $77 \%$. Em um terceiro estudo $^{(17)}$ a licença médica também representou a fatia mais significativa entre as ausências não previstas.

Comparando o IST nos dois anos estudados, observamos que esse índice tende a se manter em 2007, podendo até subir se considerarmos que a instituição reduziu os contratos temporários. Em nossa vivência, este fato relaciona-se a um ciclo vicioso que se estabelece, pois havendo número menor de contratos em decorrência da contenção de despesas, acarreta a sobrecarga da equipe fixa que precisa fazer maior número de horas extras e, conseqüentemente, se reflete em aumento de faltas e licenças médicas. Em 2006, o quadro de ausências não previstas teve sua cobertura em torno de $100 \%$ com contratos temporários, ao passo que no ano de 2007 , essa cobertura caiu para cerca de $50 \%$, por falta de recursos financeiros.

Vale ressaltar que os trabalhadores vinculados a FAMESP, não tem direito a horas extras, ficando esse direito assegurado somente para os funcionários estatutários, e isso têm sido feito de maneira sistemática nos últimos anos.

\section{CONCLUSÕES}

O absenteísmo entre os técnicos e auxiliares de enfermagem representam um grande desafio para o enfermeiro, pois este precisa garantir que o cliente seja atendido em todas as suas necessidades, e para que isso seja possível, faz-se necessário que o quadro de funcionários esteja completo e de acordo com o dimensionamento necessário.
Vários estudos vêm sendo realizados sobre absenteísmo e dimensionamento de pessoal. Todavia, ainda existem muitas dificuldades para reduzir o déficit de funcionários.

Entre os principais achados encontramos um IST cuja média gira em torno de $51 \%$. Concluímos que, embora próximo, está ligeiramente superior ao encontrado em outra realidade de Hospital Universitário. Entre as ausências não previstas, destacam-se as licenças médicas, que podem estar relacionadas à sobrecarga da equipe.

Neste contexto, destacamos que a grande dificuldade é garantir a cobertura de folgas, férias e feriados, enfim, direitos trabalhistas legais, para a cobertura dos serviços de 24 horas. Outro ponto para reflexão é que, em situação de crise, na qual se faz necessária contenção de recursos financeiros e redução da equipe, ocorre diminuição do número de contratos, como verificado neste contexto. $O$ efeito cascata que se produz é o de sobrecarga do trabalhador que desencadeia aumento de faltas e licenças médicas, sendo, portanto, questionável se esse tipo de medida administrativa reduz de fato os custos ou pode até mesmo aumentá-los pelos desdobramentos gerados por essa problemática, conforme discutimos.

Além disso, outros impactos, de difícil mensuração, podem até ficar sem registros, como os que implicam na qualidade de vida do trabalhador e, conseqüentemente, na qualidade da assistência prestada. Com ou sem crise, dificilmente há redução de leitos ou da carga de trabalho e o cliente precisa continuar sendo atendido em suas necessidades. Além disso, é bom lembrarmos que a prestação de assistência humanizada depende, não somente, mas também das condições de trabalho a que estão submetidos os trabalhadores.

Acreditamos que este e novos estudos referentes a essa problemática possam trazer benefícios e melhorias para a instituição, clientela e trabalhadores.

\section{REFERÊNCIAS}

1. Chiavenato I. Administração de Recursos Humanos. São Paulo: Editora Atlas; 1979.

2. Conselho Federal de Enfermagem. Resolução no 293/2004. Estabelece parâmetros para dimensionar - quantitativo mínimo dos diferentes níveis de formação dos profissionais de enfermagem para cobertura da assistência em instituições de saúde. Ri de Janeiro (Brasil): COFEN; 2004.

3. Fugulin FMT, Gaidzinski RR, Kurcgant P. Ausências previstas e não previstas da equipe de enfermagem das unidades de internação do HU-USP. Rev. esc. enferm. USP. 2003; 37(4):109-17.

4. Robazzi MLCC, Gir SAPE, Santos WDF, Moriya TM. Serviço de enfermagem: Um estudo sobre os 
absenteismos. Revista Brasileira de Saúde Ocupacional. 1990;69(218):65-70.

5. Reis RJ, Rocca PF, Silveira AM, Bonila IML, Giné ANI, Martín M. Fatores relacionados ao absenteísmo em profissionais de enfermagem. Rev. Saúde Públ. 2003; 37(5): 616-23.

6. Barboza DB, Soler ZASG. Afastamentos do trabalho na enfermagem: ocorrências com trabalhadores de um hospital de ensino. Rev Latinoam Enfermagem. 2003; 11(2): 117-83.

7. Silva DMPP, Marziale MHP. Problemas de saúde responsáveis pelo absenteísmo de trabalhadores em um hospital universitário. Acta Scientiarum. Health Sciences 2003;25(2): 191-7.

8. Campos LF. Dimensionamento de pessoal de enfermagem nos hospitais de Ribeirão PretoSP.[dissertation]. Ribeirão Preto: Escola de Enfermagem/USP; 2004.

9. Rogenski KE, Fugulin FMT. Índice de segurança técnica da equipe de enfermagem da pediatria de um hospital de ensino. Rev. esc. enferm. USP. 2007; 41(4): 683-89.

10. Ricardo CM, Fugulin FMT, Souza TM. Dimensionamento de pessoal de enfermagem: análise do tempo efetivo de trabalho das enfermeiras da UTI pediátrica do HUUSP. Rev. gaúcha enferm. 2004; 25(3): 357-66.

11. Matsushita MS, Adami NP, Carmagnani MIS. Dimensionamento do pessoal de enfermagem nas unidades de internação do Hospital de São Paulo. Acta paul. enferm. 2005; 18(1):9-19.

12. Gaidzinski RR. Dimensionamento de pessoal de enfermagem. In: Kurcgant, P. (org). Administração de em Enfermagem. São Paulo: Editora EPU; 2003. p. 91-6.

13. Laus AM, Anselmi ML. Caracterização dos pacientes internados nas unidades médicas e cirúrgicas do HCFMRP-USP, segundo o grau de dependência em relação ao cuidado de enfermagem. Rev Latino-am Enfermagem. 2004; 12(4):643-9.

14. Fugulin FT, Gaidzinski RR. Horas de Assistência de Enfermagem: Análise comparativa de parâmetros. Revista Nursing. 2000; 23(3):30-4.

15. Antunes AV, Costa MN. Dimensionamento de pessoal de enfermagem em um hospital universitário. Rev Latino-am Enfermagem. 2003; 11(6): 832-9.

16. Alves M. O absenteísmo do pessoal de enfermagem nos hospitais. Rev. gaúcha enferm. 1994; 15(1/2): 71-5.

17. Fugulin FMT, Gaidzinski RR, Kurgant P. Ausências previstas e não previstas da equipe de enfermagem das unidades de internação do HU-USP. Rev. esc. enferm. USP. 2003; 37(4): 109-17.

18. Gaidzinski RR, Fugulin FMT, Castilho V. Dimensionamento de pessoal de enfermagem em instituições de ensino. In: Kurcgant, P.(coord) Gerenciamento em enfermagem. Rio de Janeiro: Editora Guanabara- Koogan; 2005. p.125-37.
19. Calderero ARL, Miasso Al, Corradi-Webster CM. Estresse e estratégias de enfrentamento em uma equipe de enfermagem de Pronto Atendimento. Rev. Eletr. Enf. [Internet]. 2008 [cited 2009 mar 23]; 10(1):51-62. Avaliable from: http://www.fen.ufg. br/revista/v10/n1/pdf/v10n1a05.pdf 20. Medeiros SM, Ribeiro LM, Fernandes SMBA, Veras VSD. Condições de trabalho e enfermagem: a transversalidade do sofrimento no cotidiano. Rev. Eletr. Enf. [Internet]. 2006 [cited 2009 mar 23]; 8(2):233-40. Avaliable from: http://www. fen.ufg. br/revista/revista8 2/v8n2a08.htm

Artigo recebido em 13.12.07.

Aprovado para publicação em 25.05.09. 